\title{
Morpho-spectral imaging in the biosciences
}

\author{
Cyril Petibois • Yeukuang Hwu
}

Published online: 27 September 2013

(C) Springer-Verlag Berlin Heidelberg 2013

Three-dimensional (3D) imaging is a current challenge in biology and medicine for investigation of the smallest details of living systems, from cells to organisms, and with the expectation of micro to nanoscale resolution in real time. This is not a new objective, but rather a long quest toward excellence; to achieve such analytical performance, however, imaging technology must integrate the latest innovations in light sources, optics, detectors, supercomputers, and automation, among others.

Spectroscopic imaging has already been proved to achieve $3 \mathrm{D}$ rendering, either directly with a confocal microscope (e.g., Raman microscopy) or with post-acquisition reconstruction (e.g., infrared (IR), mass spectrometry (MS), and X-ray fluorescence (XRF) microscopy), which work in transmission mode or on the sample surface. The main advantage of the development of 3D spectroscopic imaging is to provide multiple chemical information extracted from raw spectra. The real challenge seems to be the ability to normalize the signalto-noise (SNR) ratio between 3D images stacked for 3D rendering. The duration of acquisition, the stability of light sources, and signal quantification are the three main components of SNR instability or fluctuations. It seems that the development of new sources is crucial to the development of 3D imaging technology for the achievement of micro and nanoscale resolution. At the very least, this source

Published in the topical collection Morpho-Spectral Imaging with guest editors Cyril Petibois and Yeukuang Hwu.

C. Petibois $(\bowtie)$

Université de Bordeaux, Inserm U1029, Avenue des Facultés, Bat. B2, 33405 Talence-Cedex, France

e-mail: cyril.petibois@u-bordeaux2.fr

Y. Hwu $(\triangle)$

Academia Sinica, Nano-X Laboratory, No 128, Sec. 1, Academia Road, Nankang Taipei 115-29, R.O.C, Taiwan e-mail: phhwu@sinica.edu.tw development is mandatory for introduction of $3 \mathrm{D}$ spectral imaging techniques into the routine environment of biological laboratories or hospitals for diagnostic purposes. Saving time in data acquisition and treatment to obtain a 3D image of a sample is the current major limitation for merging these $3 \mathrm{D}$ imaging techniques with commercially available industrial products. This is frustrating the expectations of the spectroscopist community wishing to exploit these new methods of spectral analysis. Nevertheless, Raman microscopy has recently benefited from low-power lasers and ultra-fast detectors, thus limiting the destructive effect of lasers on organic samples and leading to in vivo analysis of small biological specimens. IR imaging is now being proposed for this application with tunable lasers for stable SNRs with ultra-fast acquisition (ms range).

The first attempts at $3 \mathrm{D}$ rendering with these techniques have led to a glimpse of the tremendous analytical potential these approaches will bring to biosciences, notably by achieving microscopic rendering of large samples. Each technique benefits from its own physical properties, leading to different developments for specific uses in the biosciences. Raman microscopy is currently more successful for cell imaging, because of its confocal geometry and nanoscopic resolution. IR imaging has greater potential for analyzing larger samples (typically tissues) because of focal plane array detectors and rapid acquisition with high SNRs. XRF is sensitive enough to achieve trace analysis of multiple inorganic elements in cells and tissues. Laser ablation-based inductively coupled plasma mass spectrometry (ICP-MS) has also been used to achieve nanoscopic resolution for detection of trace concentrations of metals; it is, thus, also compatible with cell imaging dimensions.

All of these spectroscopic imaging techniques will soon be available for fully quantitative 3D analysis, leading to tomographic reconstruction when they are coupled to morphological imaging, notably through X-ray microscopy, which is able to reveal the microscopic structure of hard materials (e.g., 
bone, cartilage) and functional networks (e.g., vasculature, connective tissue). Such coupling between morphological and chemical imaging is also expected to reveal the relationships between microvasculature and cellular microenvironments in degenerative pathologies (including Alzheimer's disease and chronic kidney disease) and cancers (related to angiogenesis). X-ray microscopy is also being considered for multiple dimensions of the biological continuum, from the whole body (at moderate spatial resolution) toward the single cell, revealing astonishing details of cell-cell interactions, tissues, and the ultrastructures of organs. Subsequently, coupling with spectral imaging techniques will become an exceptional way to investigate biosamples, thus defining the new challenge of this field, namely, merging morphological and spectroscopic data from biosamples for 3D multimodal imaging.

Whereas mesoscopic positron emission tomography (PET) and magnetic resonance imaging (MRI) reveal diseased organs in the whole body, microscopic morpho-spectral analyses will reveal the basis of the pathology in tissues, and nanoscopic developments will complete the picture by extracting crucial molecular data for treatment. This imaging continuum will shed new light on biology and medicine by enhancing our knowledge of the links between molecular, cellular, and organizational aspects of pathologies.

The first MSIB (Morpho-Spectral Imaging in Biosciences) workshop was held in Bordeaux on the 5th and 6th November, 2012, with the ambition of laying the foundations of a large group of experts to define the steps necessary for the development of techniques enabling hybrid imaging by combining morphological approaches with molecular and chemical analysis at micro and nanoscale resolution. This first MSIB workshop was organized between the scientific communities of France and Taiwan to evaluate the potential for collaboration between a leading nation in biology and medicine and one of the leading developers of semiconductors, supercomputers, and imaging resources in the world. The workshop mixed technological and biological talks to predict future applications of hybrid imaging techniques and define a strategy for obtaining new instrumentation designed for routine use. The success of this workshop led to the announcement of a second meeting, in 2013, from 31st October to 2nd November, in Hualien, Taiwan, R.O.C. This second MSIB workshop will be more thematic, including, as the main topics, multimodal imaging, 3D analysis of pathologies, and a special session devoted to the new concept of "image processing with spectromics".

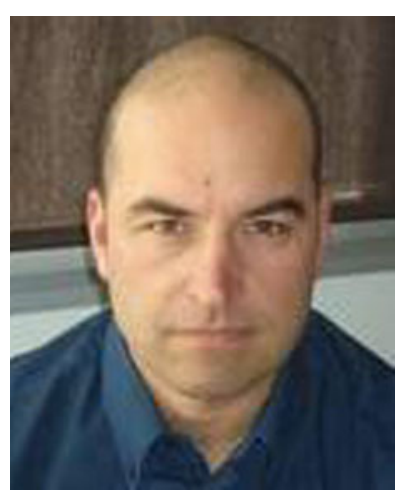

Cyril Petibois, Assistant Professor of Biochemistry at the University of Bordeaux, France, is involved in the development of imaging methodology for biosample analysis (Inserm U1029 research unit; "Biophysics of vascular plasticity" group). His research interests involve the development of multimodal imaging methods for full characterization of biosamples, from synthetic models to individual cells and small animals, by combining elemental, morphological, molecular, and chemical techniques. His laboratory is also engaged in the development of high-performance imaging techniques, with advanced industrial collaboration with companies that are leaders in the field.

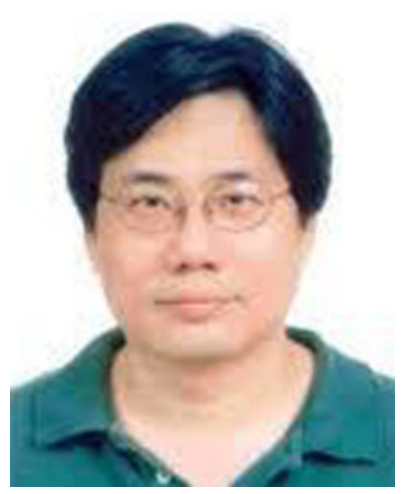

Yeukuang Hwu is a Professor of physics at the Institute of Physics, Academia Sinica, Taiwan, in charge of the NanoX laboratory. He has published more than 150 scientific papers and registered several patents related to X-ray imaging and its applications. $\mathrm{X}$ ray imaging (radiology) is the most widely used X-ray technique and his main research interest is the development of effective methods for characterization of biological objects, notably the microvascular network, in organs and tissues. In recent years, because of the excellent performance of Xray sources boosted by synchrotron radiation, X-ray microscopic imaging reached a new level of performance in resolution and contrast which, in turn, has led to breakthroughs in the nano-characterization of biosamples, from the individual cell to small animals. 